\title{
A New Strategy for Optimizing Energy and Delay in MCSMAC Protocol
}

\author{
Ali Chodari Khosrowshahi ${ }^{1 *}$, Bahman Arasteh ${ }^{2}$, Saeid Taghavi Afshord ${ }^{3}$ and Behnam Arasteh ${ }^{2}$ \\ 'Department of Computer Engineering, Islamic Azad University, Osku Branch, Osku, Iran; researchtmp@gmail.com \\ ${ }^{2}$ Department of Computer Engineering, Islamic Azad University, Tabriz Branch, Tabriz, Iran \\ 3Department of Computer Engineering, Islamic Azad University, Shabestar Branch, Shabestar, Iran
}

\begin{abstract}
MCSMAC refers to a protocol which is aimed to control multi-channel medium access in wireless sensor networks (WSNs). In this paper, we report a study in which we attempted to optimize the MCSMAC protocol. This protocol was designed on the basis of the S-MAC protocol and is considered to be a kind of contention-based protocol. Any MAC protocol which is designed for sensor networks should take the specific circumstances and constraints of such networks into account; some of the important constraints and features of them are limited resources, high density and traffic among sensor nodes and unstable state of network nodes. Wireless communications among sensors consume the majority of the energy in a network. The primary purpose of our optimization study on the MCSMAC protocol was to reduce the energy consumption and the delay time while transferring data packets. The results obtained from the simulations carried out in this study verify and acknowledge the mentioned improvements in the MCSMAC. This protocol operates efficiently in highly dense and dynamic networks. The energy consumption and delay time of this protocol were meticulously examined under different topologies and traffic loads. Based on the conducted investigations, some equations were proposed for choosing the appropriate parameters for different functions.
\end{abstract}

Keywords: Wireless Sensor Networks (WSNs), Medium Access Control (MAC), Energy, Delay

\section{Introduction}

Significant technological advances in wireless communications, energy efficient electronic equipments and radio frequencies have provided the ground for researchers to develop low-power sensor nodes for transferring, processing and sensing data wirelessly. Such tiny sensor nodes function in a self-organized manner in the network and cooperate with other nodes in order to fulfill shared tasks such as sensing industrial data, observing environment or detecting valuable equipments. In general, although different types of communication such as one-to-one correspondence between sensors exist, communication between the sensor nodes and the central location which is called the sink is established through multi-hop wireless links ${ }^{1}$. One of the main challenges in sensor networks is deemed to be the achievement of the energy efficiency in wireless channels. The extensive broadcasting nature of the wireless medium stipulates that successful communication should be maintained by means of a unique link between the transmitter and the receiver of data. Medium access protocol (MAC) is in charge of establishing such unique and individual communications in the network. MAC protocols in WSNs are different from other wireless networks because media access is critical for energy consumption in WSNs; the proposed MAC protocol for the WSN should take this parameter into consideration.

MAC protocols have been widely studied in relation to wireless sound and data communications in the related literature. There are two major classes of strategies for designing MAC protocols in WSNs which are referred to as schedule-based and contention-based strategies ${ }^{3}$. Schedule-based MAC protocols depend on time schedule and reservation. An example of this protocol (CSMA) is demonstrated in figure 1 . On the other hand, contentionbased MAC protocols draw upon contentions based on a determined mechanism to access the shared medium. An instance of this type of protocols is CSMA. In the protocol CSMA/CA, CA stands for collision avoidance. This

${ }^{*}$ Author for correspondence 
protocol has been adjusted for local wireless networks by means of IEEE $802.11^{6}$. The sequence of the RTS/CTS/ DATA/ACK packages was used in the protocol which is demonstrated in figure 2 below. Some important factors that should be considered in designing MAC protocols ${ }^{4}$ are the methods and procedures of combating the waste of energy and energy abuse; in particular, effective avoidance of collision, scalability, efficient use of a channel, delay, throughput and fairness are some important examples of those factors which should be taken into account.

Our goal for designing a new MAC protocol for WSNs is to propose a method which has significantly low energy consumption and low latency. Due to the unique and distinctive characteristics of sensor networks, it is essential and significant that typical MAC techniques be modified and revised. The first reason behind such an impetus is that traditional MAC protocols have been designed for single-hop sensors and hence are not appropriate for multi-hop communications. The second reason for the necessity of modifications and adjustments in MAC techniques is the high significance of energy consumption in sensor networks; however, in designing traditional MAC, the factor of energy consumption has not been taken into consideration. The third rationale for the modification of MAC techniques is that a sensor network is made up of hundreds or even thousands of sensor nodes and their set-up is not predetermined and preordained; as a result, it can be maintained that MAC protocol needs to self-organize and self-arrange the network. Idle listening, collision, overhearing, protocol overload and traffic changes can be considered as the main sources for the waste and misuse of energy in contention-based MAC protocols 5 . Many research studies have been conducted for designing MAC protocols and several protocols such as $\mathrm{S}^{-\mathrm{MAC}^{4}, \mathrm{MCSMAC}^{2} \text {, CL-MAC }}{ }^{7}$ and $\mathrm{COSMIC}^{9}$ have been proposed. Moreover, some efforts have been made for evaluating these protocols and their different functions and applications s,8,10, $^{5}$; the results of such evaluating research studies may be used for selecting the appropriate protocol for an application. In line with the purpose of the present study, it can be argued that the proposed protocol, among many other protocols, has considerably low energy consumption with remarkably low latency in most cases. We optimized this protocol for energy consumption and latency. A significant difference between the proposed approach (enhanced MCSMAC) and MCSMAC is that the band widths of channels in the old protocol is equal while they are different in the enhanced MCSMAC. In other words, an outstanding advantage of the proposed approach is that the size of the allocated band width in each data channel is based on the rate of use of that channel.

The scientific contributions of the present study can be listed as follows:

1. Introduction of the enhanced MCSMAC as an optimization for MCSMAC.

2. Reduction of energy consumption in a WSN.

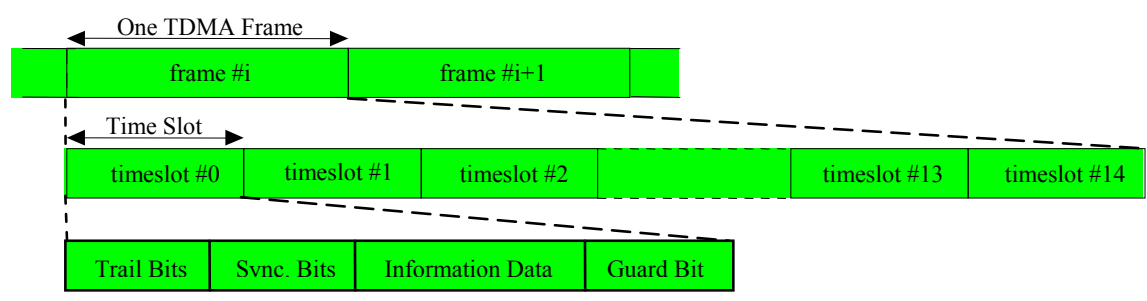

Figure 1. TDMA connection: in each frame, one time unit is allocated to each group. TDMA is originally collision-free

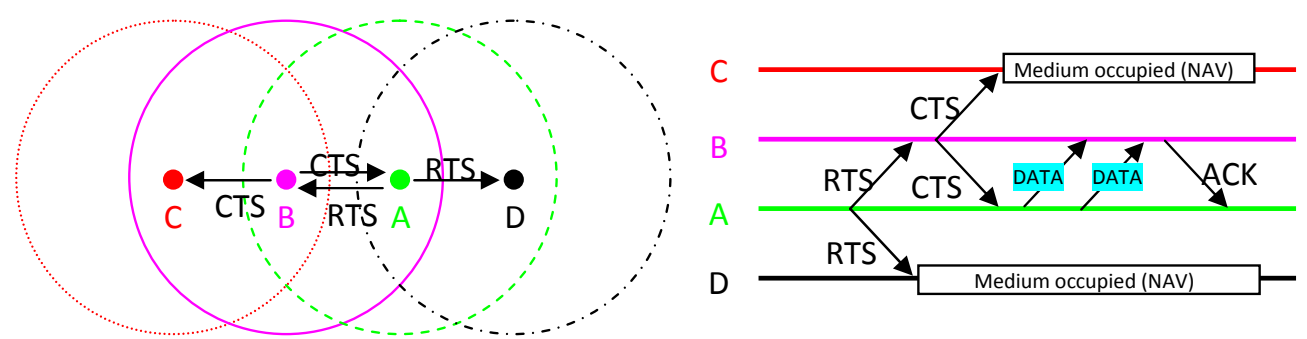

Figure 2. CSMA connection: data transition is carried out as a follow-up to RTS/CTS exchange in order to reduce the probability collision 
3. Reduction of latency

4. Differentiation in the size of band widths as a function of the rate of the use of that channel.

The remainder of this paper is organized as follows. In Section 2 we present an overview of MCSMAC. In Section 3 the conducted optimization on the MCSMAC protocol is described. Section 4 reports the simulation results with evaluations of the performance. Finally, Section 5 concludes the paper.

\section{An Overview of MCSMAC}

MCSMAC is a kind of MAC protocol which has been designed for wireless sensor networks (WSNs) ${ }^{2}$. This protocol is considered to be a contention-based protocol. Designing this protocol was based on S-MAC. The protocol is intended to reduce energy wasting factors such as those mentioned above. The following section introduces methods with which MCSMAC has tried to reduce the waste of energy in sensor nodes.

\subsection{Periodical Listening and Sleeping}

In sensor nodes, processing, sensing and wireless connection account for the majority of energy consumption out of which wireless connection has the most energy consumption. Hence, one main energy wasting component is the transceiver of sensor nodes, especially when it is idle and does not perform a task ${ }^{4}$. One possible solution to this problem is to turn off the radio when it is not necessarily required. In MCSMAC, in order to reduce energy consumption, the radio will be turned off in its idle times and the sensor node will go into sleep position; this procedure is performed based on the scheduling plans. Figure 3 demonstrates these changes in the modes of sensor nodes which are conducted periodically and in line with scheduling plans.

The entire cycle of listening and sleeping is called a frame. The proportion of listening interval to the whole length of frame demonstrates the active and working cycle of the frame. The sleeping interval can indeed differ in different applications and hence it is variable and in fact can affect the working cycle.
Since the radio is completely turned off in the sleep mode, as a result, there will be no possibility of communication for the node and it will be unaware of any communications and it will be able to communicate in its awake and active mode. One more problem is that a node which wants to send information to another node has no idea of whether the target node is in the sleep mode or the active mode. It is likely that when the target node is in sleep mode, the source node is in the awake mode or when the target node is in the awake mode, the source node is sleeping; under these circumstances, maintaining communication between the nodes will not be possible. In order to solve this problem, sensor nodes must coordinate and synchronize their sleep and awake modes with one another and function in congruity. This task is fulfilled in the synchronization interval. The synchronizing procedures have been detailed in ${ }^{2}$.

All the sensor nodes are self-governing and free in scheduling and planning their sleep and awake intervals. The nodes share their scheduling with each other through a periodical comprehensive distribution of a SYNC packet with their direct neighbors. The interval in which a SYNC packet is sent is referred to as a synchronization interval. Although the task of periodic sleep reduces the consumption of energy, periodic sleep increases the delay time. In [4], a mechanism, known as adaptive listening, has been described in S-MAC which is intended to reduce delay. Such a mechanism is also applicable for MCSMAC.

\subsection{Collision Avoidance}

In case several neighboring nodes plan to send information while listening, it might result in the occurrence of collision and hence the sent packets will be destroyed. Such a condition leads to the wasting of energy. Under these circumstances, the nodes will be required to compete with one another to send their packets. Contention-based protocols such as IEEE 802.11, S-MAC and MCSMAC use similar methods to avoid collision ${ }^{2,4,6}$; these protocols have utilized virtual and physical carrier sensing and RTS/CTS exchanges for the problem of hidden terminals. In such protocols, a sequence of RTS/CTS/DATA/ACK is used for the transition of data.

\begin{tabular}{l|l|l|l|l|l}
\hline$\cdots$ & Listen & Listen & Sleep & $\cdots$ \\
\hline \multicolumn{2}{c|}{ time }
\end{tabular}

Figure 3. Periodical listening and sleeping 
In addition to the collision avoidance mechanism, MCSMAC has also employed a frequency division method. In MCSMAC, communication band width has been divided into several channels. One of those channels is known as a control channel and the controlling channels RTS/CTS are sent and received through this channel (channel zero). Depending on the type of application and the purpose, the number of channels can differ. Indeed, the number of channels is one of the parameters of MCSMAC. Data packets are sent and received in a distinct channel of control channel. Since the number of channels is limited for the flow of sensor nodes, the neighboring nodes compete with one another to obtain the channel. The existence of many channels is considered as an advantage since several neighboring nodes can communicate with each other at the same time; however, the band width utilization can decrease in this case. Hence, the number of channels should keep a balance, that is, depending on the traffic circumstances and the number of neighbors (aggregation of nodes), the number of channels should be selected.

In order to select the channel for data transmission, the source node selects a free channel and inserts it in the RTS packet and sends it to the destination. After receiving the RTS packet, destination node examines the suggested channel and in case it has no problem, the destination node inserts the number of channel in CTS packet and sends it to the source node. Otherwise, another channel is selected and the source node is informed of the selected channel by CTS packet. The mechanism of channel selection for transmitting data has been completely described in [2]. The difference of MCSMAC with 802.11 and S-MAC is that in 802.11 and S-MAC only one packet can be sent at a time in one neighborhood but in MCSMAC more than one packet can be transmitted. The number of flows depends on the number of channels. Figure 4 demonstrates the method of access to channel in MCSMAC.

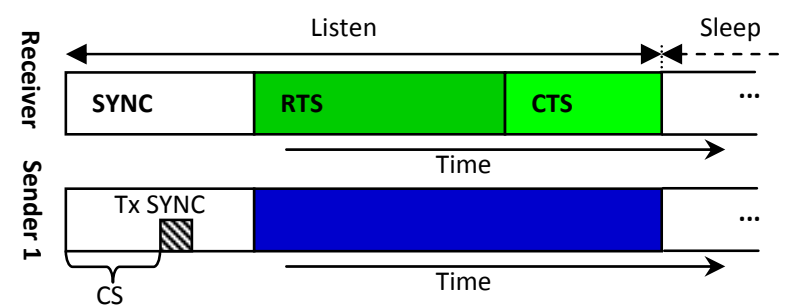

\subsection{Sleeping Coordination}

As discussed above, each sensor node will have a sleep interval and a listen interval. The transition of RTS and CTS packets is carried out in the listen interval. Each node goes to the listen and sleep states in its schedule; due to this, each node should select a schedule for itself and inform the neighbors. Moreover, nodes periodically use the SYNC packet in order to synchronize their sleep and listen intervals. Each node is required to adopt a schedule and exchange it with its neighbor nodes before it can start periodic listen and sleep operations. Each node maintains a schedule table that stores the schedules of all its known neighbors. Figure 5 shows the scheduling in the receiver and different types of senders. As it is demonstrated in Figure 5, sender 1 only sends a SYNC packet; sender 2 only sends a uni-cast data packet and sender 3 sends both SYNC and data packets. This process is fully explained $\mathrm{in}^{2}$.

\subsection{Avoidance of Idle Listening}

When a sensor node has to listen to the packets being sent to gain information about the present transmissions, redundant listening takes place. Contention-based protocols have lower energy efficiency than TDMA protocols. This is more evident in 802.11 where each node has to listen to all the neighboring transmissions to perform virtual carrier sense; as a result, each node listens to many packets, even if it is not directly sent to it. This task wastes a significant amount of energy, in particular when the aggregation of the nodes and the traffic jam are high.

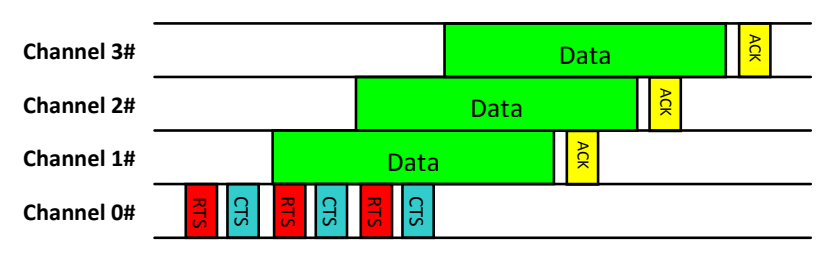

Figure 4. The channel accessing method in MCSMAC

Figure 5. Timing relationship between a receiver and different senders. CS stands for carrier sense. 
MCSMAC is intended to avoid redundant listening by means of putting the node in the sleep mode. Data packets are usually much longer than the control packets. In MCSMAC, the transmission of RTS/CTS control packets is done via control channel. There is a NAV field on CTS/RTS. Each node keeps NAV in order to specify the activities of its neighbor and determine their engagement and busy state. When a node receives a RTS/CTS packet which was specified for another node, it updates its NAV array by means of the length field and the channel field. Any value of NAV beyond zero shows that an active transmission has been conducted on the channel in the neighborhood. As the timer of NAV starts the counting, the value of NAV decreases continuously. Thus, a node will go into sleep mode in order to avoid redundant listening if the NAV value is beyond zero. Also, if the NAV value of the control channel is not zero, the node will sleep until it becomes zero.

One more issue relates to the transmission of big masses of data packets. A shortcoming of transmitting big data packets as individual packets is the cost of transmitting them for a second time in case they are broken. In $^{2}$ a method has been introduced which splits big data packets into smaller packets and the selected channel remains reserved until all the packets are transmitted and the reservation may not be extended and the relevant node does not have to make use of RTS/CTS for each packet; this packet is sent once at the beginning which reduces the amount of energy consumption.

\section{The Conducted Optimization in this Study}

In simulating MCSMAC, we planned to check all the channels from lowest numbers to highest numbers successively in order to select the proper channel. In other words, if channel number 1 is not vacant, channel 2 will be checked. As a result of analyzing simulation results, we realized that the degree of application of channel number zero which is a control channel is more than the other channels. As the number of channels goes up from zero to higher numbers, the amount of their application and utilization decreases. Hence, we discovered that it is not necessary to se the same amount of band width for all the channels. It is obvious that a channel with high probability of use and application should have more band width.

In case $\mathrm{N}$ stands for the number of data channel and $\mathrm{B}$ stands for the band width for data channels, the proposed band width for each data channel $\left(\mathrm{B}_{\mathrm{i}}\right)$ will be as below if $\mathrm{i}=1,2, \ldots, \mathrm{N}$ :

$$
B_{i}=\frac{B}{2^{i-1} \times\left(2-\frac{1}{2^{n-1}}\right)}
$$

It should be noted that this is a proposed value. We made use of this condition in our simulations.

As it has been shown in figure 4 above, if the same band width is to be used for data channels, the time length of transmitting data will be the same in all of the channels, that is, their NAV value will be the same. However, if the band width of the data channels is different, their NAV will also be different. Figure 6 demonstrates the method of data exchange.

As the figure above shows, the band widths of those channels which are highly likely to be used are much bigger in the figure. Indeed, the size of channel will be a function of the probability of the selection of the channel for transmitting a packet.

\section{The Experiment}

The purpose of this experiment is to reveal the equilibrium and balance of energy and delay in MCSMAC. In order to make comparisons, we simulated the different models of MAC and then examined them on different topologies and measured their efficacy in terms of energy consumption and delay. In ${ }^{2}$, the results of experiments have been analyzed on different models like S-MAC, MCSMAC, SMACS and protocols such as IEEE 802.11; for this reason we did not mention the results of the experiment in this article. In this section, the results of the experiments on protocols of S-MAC, MCSMAC and the optimized MCSMAC are reported for the sake of comparison. In this study, we focused on the factors of delay and energy consumption. The efficiency of these protocols was examined under three different topologies

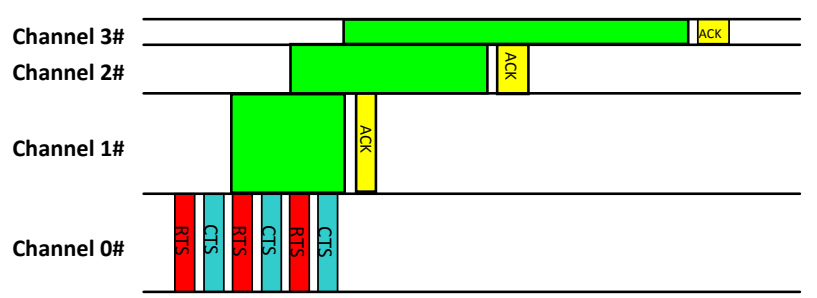

Figure 6. The channel accessing method in optimized MCSMAC 
of 1-hop, 2-hop and 3-hop. The results of examining the efficiency are mentioned below. In each of these conditions, data packets are transmitted from the source node to the target node in different time intervals.

\subsection{Measuring Energy Consumption}

In order to measure the energy consumption of the radio, we considered three modes for the transceiver, that is, sleep, awake (idle), transmission and receiving. Each of these modes has a different amount of energy consumption. In the experiment, we measured the length of time during which the radio of each node was in those modes. Then, we measured the consumption of energy by means of multiplying the obtained time by the required power of each mode and adding them together. We considered the energy consumption of the awake mode as 1 , of transmission as 1.4, reception as 1.05 , and sleep as 0 . If $\mathrm{A}_{\mathrm{i}} \mathrm{i}$ equal to the sum of times of radio in the awake state in node $i, T_{i}$ is the sum of times of radio in transmission state in node $i, R_{i}$ is the sum of times of radio in reception state in node $i$, $\mathrm{S}_{\mathrm{i}}$ is the sum of times of radio in sleep state, and $\mathrm{N}$ is the number of nodes which cooperate in the simulation, the average energy consumption will be as below:

$$
E=\frac{\sum_{i=1}^{N}\left(A_{i} \times 1+T_{i} \times 1.4+R_{i} \times 1.05+S_{i} \times 0\right)}{N}
$$

\subsubsection{The Experiment on 1-Hop Networks}

In the topology of the 1-hop network, 8 nodes have been used where all of them are located in the radio range of one another. In this network, there are 4 source nodes and 4 target nodes. Figure 7 demonstrates the topology of this network.

The experiment was conducted under differing traffic loads where the traffic load was modified by means of inter-arrival time of different messages. The purpose of

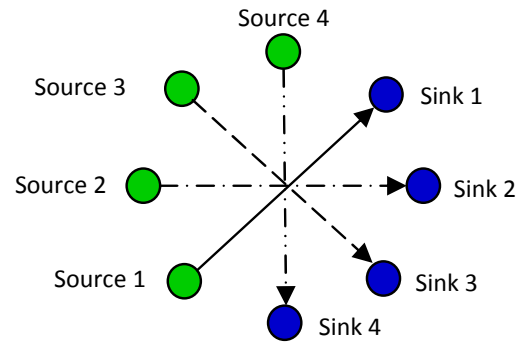

Figure 7. The topology of a one-hop network with four source nodes and four target nodes the experiment was to evaluate and measure the energy efficiency of the nodes under dense and closely packed conditions. If the inter-arrival time of a message is equal to i seconds, it means that during i seconds, one message is generated by the source node. In order to modify and change the traffic load, we carried out 10 distinct experiments by modifying the inter-arrival time from one to ten. Through the administration of these experiments, we were able to measure and calculate the energy consumption of the protocols of SMAC, MCSMAC, and the improved MCSMAC. Figure 8 demonstrates the results of experiments. In these experiments, the agents were boarded and embedded on four source nodes. In this simulation, agents typified UDP and the traffic typified CBR. Depending on the level of traffic load, each agent transmits 100 messages to their destinations and the size of each of those messages is 40 bytes.

In the conducted simulations for MCSMAC, we considered three channels (one control channel and two data channels). The band widths of the control channel one, channel zero and channel two were $\% 40, \% 40$, and $\% 20$ in proportion to the wireless medium. According to the results of the experiments, this method indicated a range of 7 to 25 percent improvement and optimization in the efficiency of energy.

\subsubsection{The Experiment on 2-Hop Network}

The topology and structure of the 2-hop network with two source nodes and two target nodes is shown in figure 9. In the demonstrated topology in the following figure, target nodes produce two data flows where both of them flow by means of the node $\mathrm{C}$. Ten traffic patterns (under different inter-arrival intervals) were independently experimented. The agents of this simulation were similar to those of the

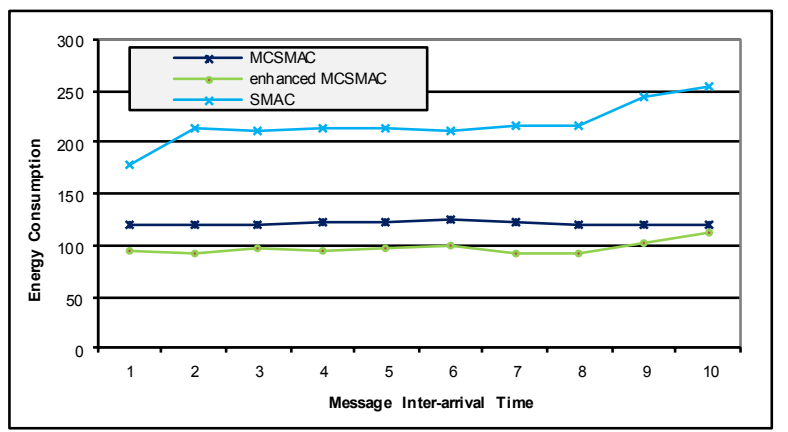

Figure 8. The average energy consumption on radios in a one-hop network 
former simulation and the collisions of the data were not taken into account.

In any test, each source node produces 200 data packets where the size of each packet is 40 bytes; data packets are transmitted from source nodes towards the target nodes at different time intervals. We measured and calculated the required energy consumption for the radio to transmit a certain amount of data by means of each node. In the total of the conducted experiments for MCSMAC and SMAC, the $\% 10$ work cycle was used.

Figure 10 indicates the average rate of energy consumption for each of the nodes in the network. The traffic load was heavy when the inter-arrival time was short. As the inter-arrival time increases, traffic load increases too. Energy consumption at different traffic loads can be in figure 10.

\subsubsection{The Experiment on Multi-Hop Networks}

The topology and structure of the multi-hop networks is made up of nodes. Figure 11 demonstrates the structure of such a network. The first and the last nodes of the network are the source and target nodes, respectively. Like the experiments reported above, we changed the traffic

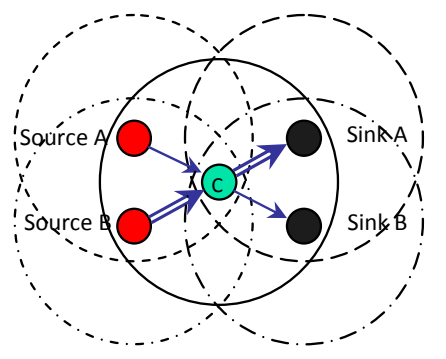

Figure 9. Topology of a 2-hop network with two source nodes and two sink nodes

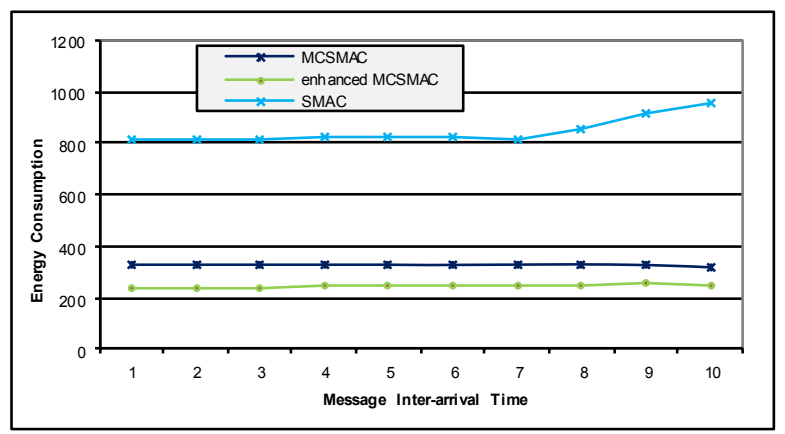

Figure 10. The average energy consumption on radios in a two-hop network load by means of modifications in the inter-arrival time of the packet in the source node. Under any traffic load, the source node transmits twenty messages where the length of each message is 100 bytes. We considered a $\% 10$ work cycle for MCSMAC and SMAC.

Figure 12 indicates the average of calculated energy consumption on the radio which is required over the entire network for transmitting a certain amount of data from the source node to the target node. As the figure below depicts, this method reduces the energy consumption by an average amount of $\% 15$.

\subsection{Calculating the Delay of End-to-End}

We used the same topologies and the same conditions of the above-mentioned experiments in order to study and examine the delay time, In order to calculate the delay time, we used the topology of the networks as those depicted in figures 6, 8 and 10 and also applied exactly the same traffic conditions. As a matter of fact, we calculated both the energy consumption and the delay time. Figures 12,13 and 14 indicate the calculated average delay time under different conditions for different protocols.

The results of the experiments revealed that the optimization and improvement fulfilled through this study also helped to reduce the delay time in MCSMAC. Indeed, delay time and the energy consumption are correlated

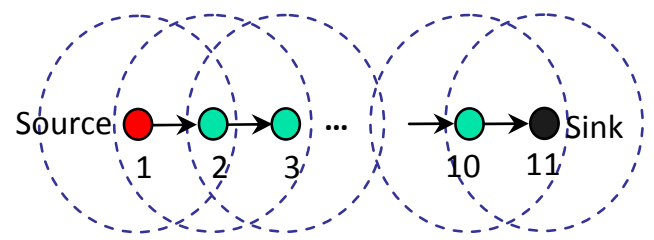

Figure 11. A 10-hop linear network with a source and a target node

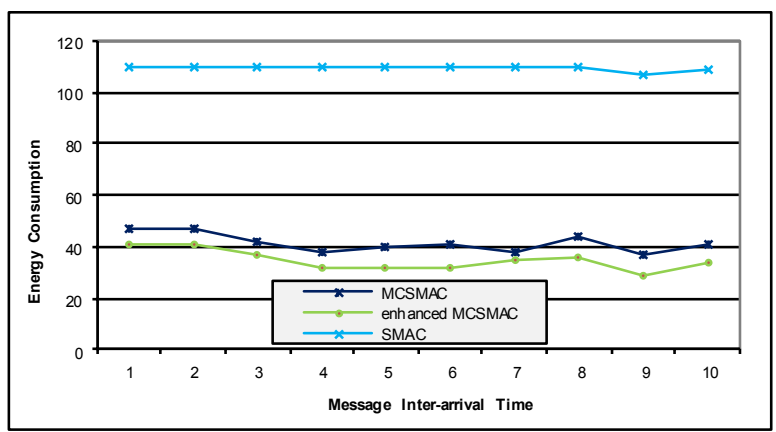

Figure 12. The average energy consumption of the radios in a 10 -hop network 


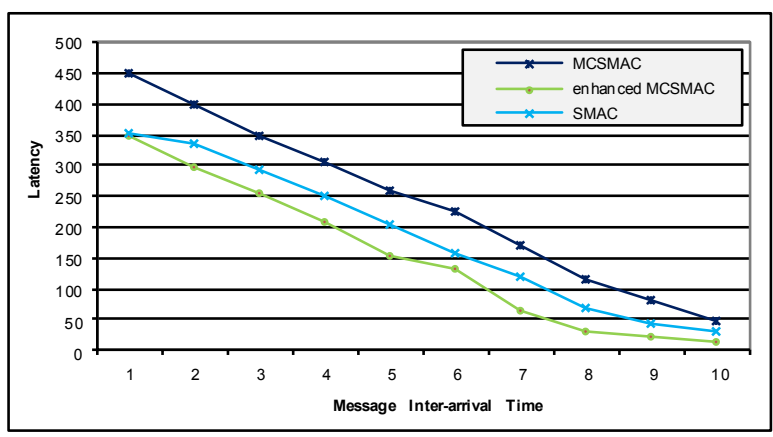

Figure 13. The average delay of message for a packet in the 1-ho network

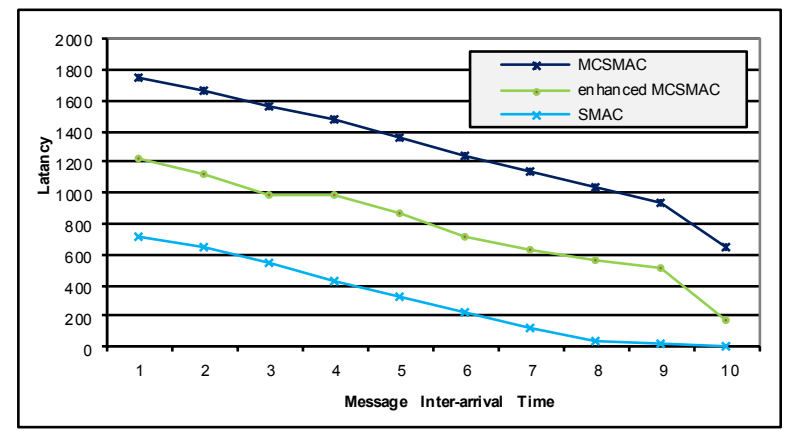

Figure 14. The average delay of message for a packet in the 2-ho network

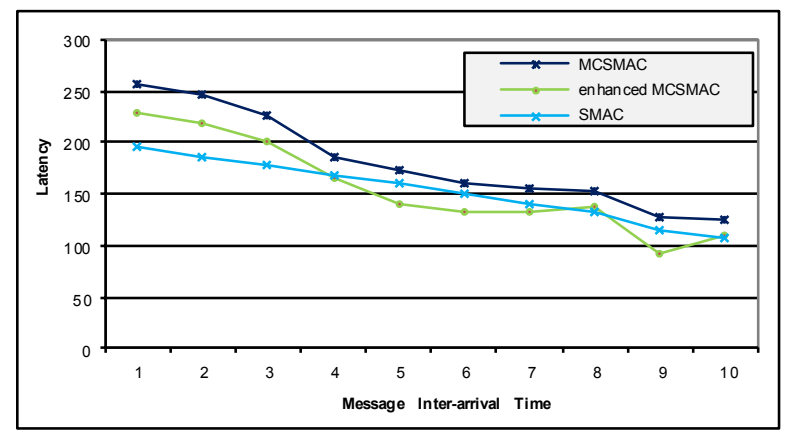

Figure 15. The average delay of message for a packet in the 10-ho network

to each other so that reducing the energy consumption increases the delay time of the network; that is why under some conditions MCSMAC has more delay than SMAC. The improvement of MCSMAC led to the reduction of the delay time to the point that in some cases MCSMAC has less delay time than SMAC. In the 10-hop network, the efficiency of SMAC under heavy traffic load is better than the improved MCSMAC; wider band is regarded as the justification and reason beyond the high efficiency of SMAC. Wider band is considered to be a factor which helps to reduce the delay time under heavy traffic for SMAC in 10-hop networks. Furthermore, in the 2-hop network, the improved MCSMAC has less delay time than MCSMAC; however, its delay is more than SMAC. The reason is the presence of a shared node between two data flows. Also, in the 1-hop network, as it has been shown in Figure 13, the improved MCSMAC reduced its delay time so that it was less than the normal MCSMAC and it was even less than S-MAC; the reason for it was the possibility of creating simultaneous communications that the neighboring nodes were able to maintain and these simultaneous communications in 1-hop networks account for the reduction of delay.

\section{Conclusion}

The contribution of this paper was an optimization and improvement for the MCSMAC in terms of delay and energy consumption. We simulated enhanced MCSMAC in NS2 and evaluated its performance in single-hop and multi-hop networks. The results of the experiments indicated a considerable improvement. Compared with other representative energy efficient MAC protocols, enhanced MCSMAC achieved the lowest duty cycle and smallest packet delivery latency. The reported improvement in delay time and the energy consumption was achieved through changing the channels and selecting the appropriate size based on the probability of their choice. As it was mentioned in [2], in terms of energy consumption, MCSMAC is more optimized and efficient than SMAC; however, in terms of delay time, SMAC is more desirable than MCSMAC. The demonstrated optimization reported in the paper helped to compensate for the weakness of MCSMAC and reduce its delay under most conditions. The only condition under which the delay time of MCSMAC is more than SMAC relates to Figure 7 where one node is shared among several data flows; however, the probability of occurrence of this condition is weak since one of the peculiarities sensor networks is the high density of the nodes and when the density is high the probability of the presence of a shared node among several flows is weak. 


\section{References}

1. P. Coronel, R. Doss, and W. Schott, "Geographic Routing with Cooperative Relaying and Leapfrogging in Wireless Sensor Networks," 2007, 646-651.

2. C. Khosrowshahi, M. Dehghan, H. Pedram, and M. Alizadeh, "A Novel Multi Channel Sensor Network MAC Protocol," 2009, 230-235.

3. J. F. Kurose and K. W. Ross, Computer Networking: A TopDown Approach, 5th ed. Addison Wesley, 2009.

4. W. Ye, J. Heidemann, and D. Estrin, "Medium Access Control With Coordinated Adaptive Sleeping for Wireless Sensor Networks," IEEE/ACM Transactions on Networking, 2004;12, 493-506.

5. F. Akyildiz, W. Su, Y. Sankarasubramaniam, and E. Cayirci, "Wireless sensor networks: a survey," COMPUTER NETWORKS, 2002;38, 393-422.

6. Wireless LAN Medium Access Control (MAC) and Physical Layer (PHY) Specification, IEEE Std. 802.11

7. A. Ben Nacef, S.-M. Senouci, Y. Ghamri-Doudane, and A.-L. Beylot, "A Cooperative Low Power Mac Protocol for Wireless Sensor Networks," in 2011 IEEE International Conference on Communications (ICC), 2011, 1-6.
8. N. Sazak, I. Erturk, E. Koklukaya, and M. Cakiroglu, "An energy efficient MAC protocol for cluster based event driven WSN applications," in 2010 International Conference on Software, Telecommunications and Computer Networks (SoftCOM), 2010,76-81.

9. A. B. Nacef, S. Senouci, Y. Ghamri-Doudane, and A.-L. Beylot, "COSMIC: A Cooperative MAC Protocol for WSN with Minimal Control Messages," in 2011 4th IFIP International Conference on New Technologies, Mobility and Security (NTMS), 2011, 1-5.

10. C. Antonopoulos, A. Prayati, F. Kerasiotis, and G. Papadopoulos, "CSMA-MAC Performance Evaluation for WSN Applications," in Third International Conference on Sensor Technologies and Applications, 2009. SENSORCOMM '09, 2009, 13-18.

11. Dong Wen, Chen Zhi-jiang, and Li Xiu-mei, "Research progress on MAC protocol for wireless sensor network," in 2011 International Conference on Consumer Electronics, Communications and Networks (CECNet), 2011, 4379-4382. 